\title{
New Manufacturing Process and Product Developments in Metal Forming Industry Using Finite Element Modelling and Optimization Techniques
}

\author{
Van Bac NGUYEN ${ }^{\mathrm{a}, 1}$, Martin ENGLISH ${ }^{\mathrm{b}}$ \\ ${ }^{a}$ College of Science and Engineering, University of Derby, Derby DE22 3AW, UK \\ ${ }^{\mathrm{b}}$ Hadley Group Technology, Hadley Industries plc, Smethwick, B66 2PA, UK
}

\begin{abstract}
The objective of this paper is to outline a practical approach using numerical modelling and optimization techniques for process and product developments in metal cold rolled forming industry. The optimum economic viability in manufacturing industry requires a minimization of the amount of material used while the structural performance of a cold roll formed product relies on maintaining the stiffness and strength of the section in applications. This leads to the development of new cold forming processes and alternative cold roll formed profiles searching for the optimal profile. In this paper, a Finite Element modelling approach was utilized to simulating complicated manufacturing process and products and optimization techniques including Design Of Experiments was used to optimize the shape design of the end products to obtain lighter products while maintaining the product strength. These developments were illustrated through two case studies of Hadley Industries plc which included (1) numerical modelling of a novel Ultra STEEL ${ }^{\circledR}$ cold roll forming process, and (2) optimization of cold roll forming sections.
\end{abstract}

Keywords. Metal forming, cold roll forming sections, Design Of Experiments, Finite Element modelling, Optimization.

\section{Introduction}

In metal forming industry, there is a critical requirement that is to reduce the initial strip to a minimum while maintaining the structural performance of the roll formed products, thus minimizing the major financial outlay in the process which is the material cost. The development of various alternative cold rolled formed profiles which improve the structural performance of the section by including additional bends such as intermediate stiffeners or ribs [1], as shown in Figure 1, has been a solution for these conflicting requirements. In addition, the development of a novel manufacturing process namely Ultra STEEL ${ }^{\circledR}$ dimpling process has been found to improve the material and structural performance by imparting a dimple plastic deformation to the whole sheet $[2,3]$, as illustrated in Figure 2.

\footnotetext{
${ }^{1}$ Corresponding Author. vb.nguyen@derby.ac.uk
} 


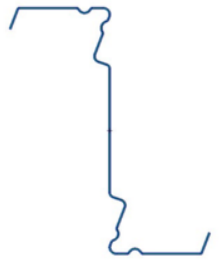

(a)

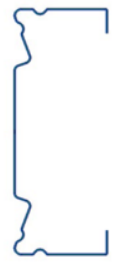

(b)

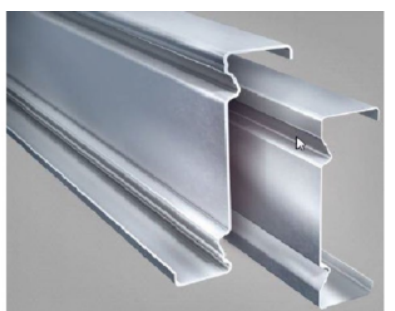

(c)

Figure 1. Intermediate stiffeners in (a) zed, (b) channel and (c) 3-dimensional profiles.
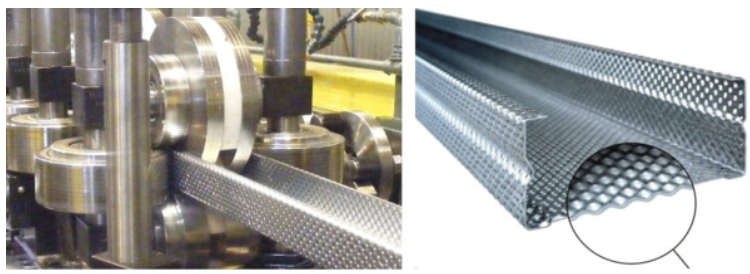

Figure 2. The Ultra STEEL ${ }^{\circledR}$ dimpling process and subsequent dimpled products.

Owing to the complex and interrelated nonlinear changes in contact, geometry and material properties that occur in the process and product forming, theoretical and design calculations cannot be used to accurately analyze the performance of both the dimpling process and products or plain products with additional bends. These, however, can be solved by using a Finite Element modelling approach which can simulate complicated processes and products [4,5]. This allows optimizing the process and subsequent products to improve the product structural performance or reducing the product material.

In this paper, Finite Element simulations and optimization using Design Of Experiments method were presented as tools for new process and product developments and illustrated through case studies of optimization of cold roll formed products of Hadley Industries plc. They include the novel Ultra STEEL ${ }^{\circledR}$ dimpling process and optimization of cold roll forming products.

\section{Simulation of the Ultra STEEL ${ }^{\circledR}$ dimpling process}

\subsection{Dimpling process}

Finite Element simulations were conducted using MARC (MSC Software, version 2019). The simulations were carried out in three stages: (1) the dimpling process was simulated first using rotating rolls, that deforms a flat steel strip into a dimpled strip, (2) the process of forming the subsequent dimpled sheet to a dimpled product using the traditional cold rolled forming process, and (3) simulating the dimpled products subject to mechanical tests for testing or practical applications. Material data obtained from physical tensile tests were incorporated into nonlinear simulations. Detailed FE model setups are described in [4-8]. In the simulation of the dimpling process, product forming, tensile and plate bending tests, 3-dimensional solid elements were used. In modelling column compression tests, shell elements were employed instead of 3-dimensional elements. FE model of the dimpling process in which the plain strip is deformed into the dimpled strip, 
is shown in Figure 3. The plain strip had a thickness of $0.90 \mathrm{~mm}$ and was modelled as a deformable body using 3-dimensional eight-node hexahedral elements. The minimum element size in the model was $0.17 \mathrm{~mm}$, small enough to model the curved geometry of the dimples.

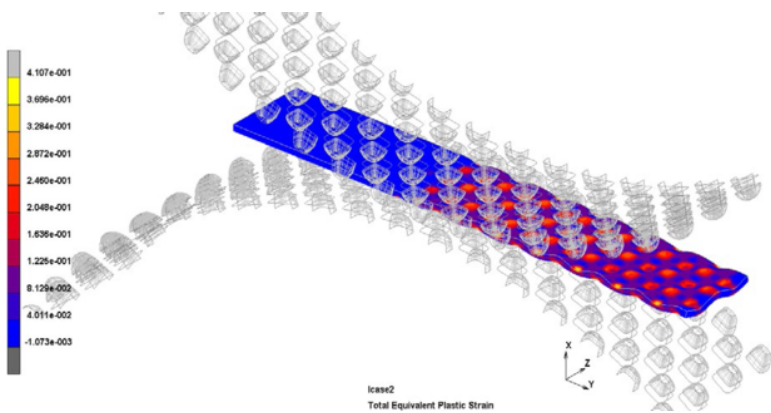

Figure 3. FE model of the dimpling process and generic dimpled strip geometry. Plastic strain contour is presented in the deformed sheet with lighter color indicate greater plastic strain magnitudes.

\subsection{Product forming process}

The cold roll forming of a dimpled product from the subsequent dimpled strip is illustrated in Figure 4. During the roll forming process, further plastic deformation was developed, especially at the section corners, gaining the maximum values at the end of the process.

(a)

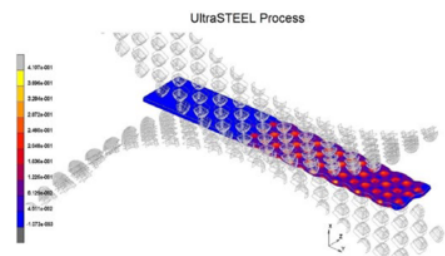

(c)

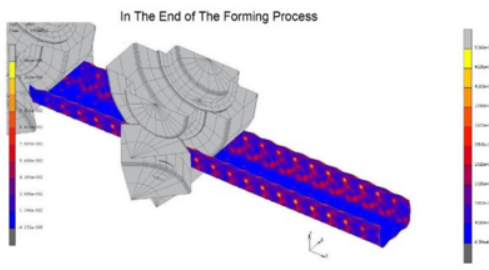

(b) in The Muddele of The Forming Processs

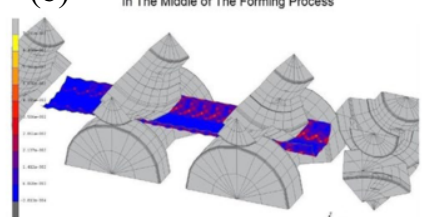

(d)

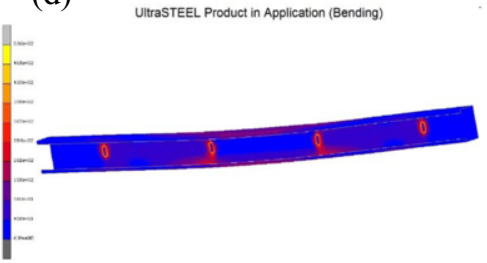

Figure 4. FE models of the dimpling process and the subsequent dimpled products (a) The Ultra STEEL process, (b) at the middle of the process, (c) at the end of the process and (d) final dimpled product.

\subsection{Product testing and simulation}

FE and experimental results of tensile, bending and column compression tests of plain and dimpled sheets are shown in Figures 5. The buckling and failed mode shapes of the dimpled column are shown in Figure 6, in which the experimental shapes were also illustrated. The FE predicted values were in very good agreement with the experimental values, with a maximum difference of $10 \%$ for all simulations. The increase in strength of the dimpled specimens is a result of the plastic deformation developed throughout the 
thickness of the dimpled sheet, during the dimpling process. The comparisons between test and simulation results demonstrate that the new Ultra STEEL ${ }^{\circledR}$ process and products were accurately represented by FE simulations.
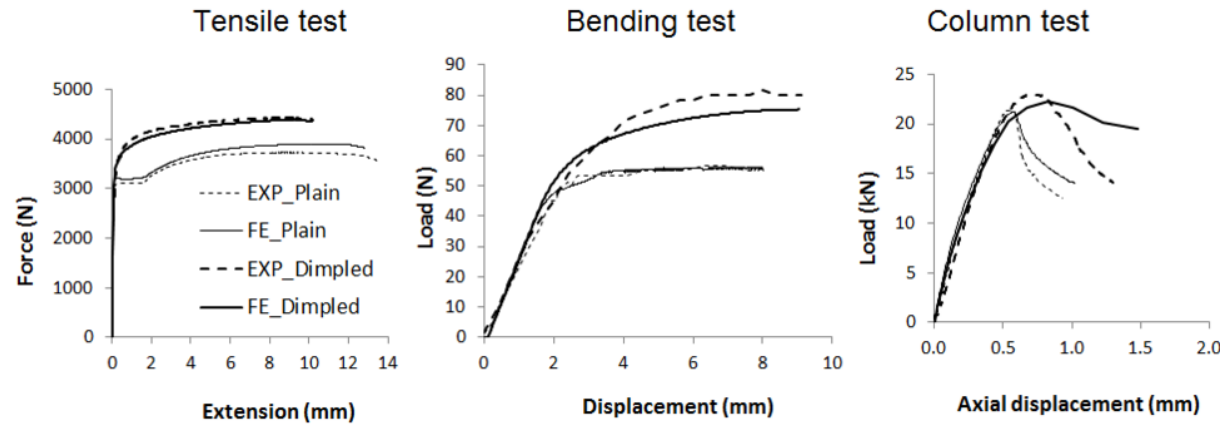

Figure 5. FE and experimental curves of tensile, bending and column tests.
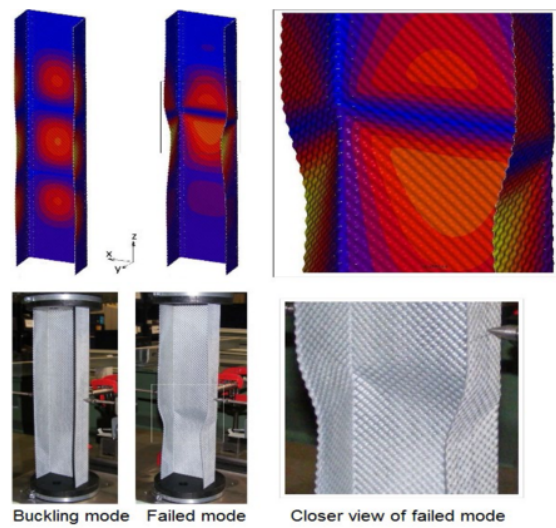

Figure 6. Buckling and failed mode shapes of the dimpled columns.

\section{Optimization of cold roll forming products}

The optimum design of a cold rolled forming product is achieved by increasing the product's strength while retaining the amount of material used. The simulations were carried out in three stages: (1) Developing new product geometries from a proposed / existing ones by varying their geometric parameters, against the target performance of the product, using parametric modelling technique via the finite element package PATRAN (MSC Software), (2) Planning the Design Of Experiments (DOE) using a response surface model, running multiple simulations, recording the performance of the system at each run and determining geometric values that give the target performance: a maximum strength to weight ratio - these procedures are done using ADAMS/INSIGHT and MARC solver (MSC Software), and (3) Simulating the mechanical tests of new products and comparing with recently conducted product test results for validation. The two case studies of this FE and optimization techniques included the design and development of new cold roll forming sections (channel). 


\subsection{Finite Element analysis and validation}

The original shape of the channel had no stiffeners and the new channel had two stiffeners positioned at an equal distance to the web center, and one stiffener on each flange positioned closely to the web-flange corner, as shown in Figure 7(a). The section has a web depth of $170 \mathrm{~mm}$, a flange width of $63 \mathrm{~mm}$ and a thickness of $1.60 \mathrm{~mm}$. The web stiffeners had a height of $20 \mathrm{~mm}$ (Dim D) and a width of $9 \mathrm{~mm}$ (Dim A); the flange stiffener had a radius of $5 \mathrm{~mm}$. The steel material has a yield stress of $519 \mathrm{~N} / \mathrm{mm}^{2}$ and a tensile strength of $550 \mathrm{~N} / \mathrm{mm}^{2}$. The position of the two stiffeners influences the channel's strength in a 4-point beam bending test. Braces at close gap $(300 \mathrm{~mm})$ were used to ensure local buckling occurred in the beams (Figure 7(b)). The failed mode shape of the channel is shown in Figure 7(c), in which the experimental shapes were also illustrated for validation. The comparisons show excellent agreements between simulation and test.

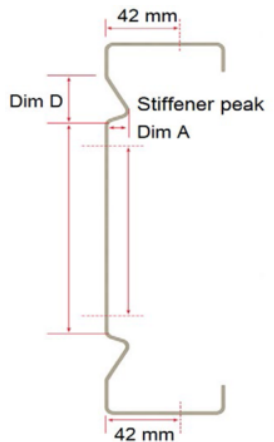

(a)

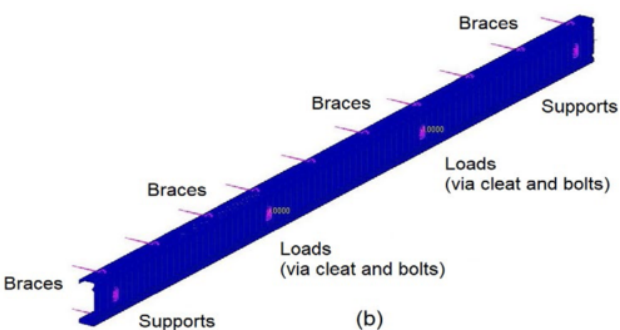

(b)

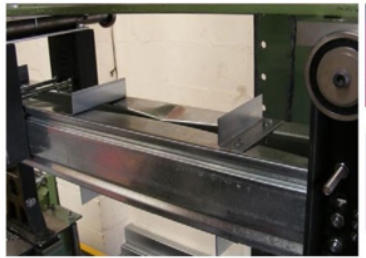

(c)

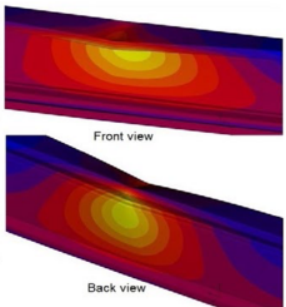

Figure 7. FE model of a 4-point bending test setup and validation including (a) cross section, (b) mesh and boundary conditions and (c) failed mode shapes of the channel section in testing and simulation.

\subsection{Finding optimal design profile}

The influence of both the web and flange stiffeners' positions, shapes, sizes, and enhanced material properties at corners and stiffeners' bends on the section's ultimate strengths was carried out. The validated FE models were utilized for the optimization study in which section dimensions were used as input parameters and the corresponding FE results (under the same applied load) were arranged in orders so that all the maximum positive effects on the section ultimate strengths were obtained, leading to an optimal design of the sections. The channel sections together with their bending setup used in the experimental testing in Section 3.1 were defined as "reference" sections while the sections without stiffeners were defined as "standard". The section length $L$, height $h$ and thickness $t$ were fixed. Figure 8 shows the ultimate moment capacities $\left(\mathrm{M} / \mathrm{M}_{\mathrm{y}}\right)$ of optimized sections (Candidates 1-6) against those of standard and reference sections. From this, the optimal design section could be obtained. 


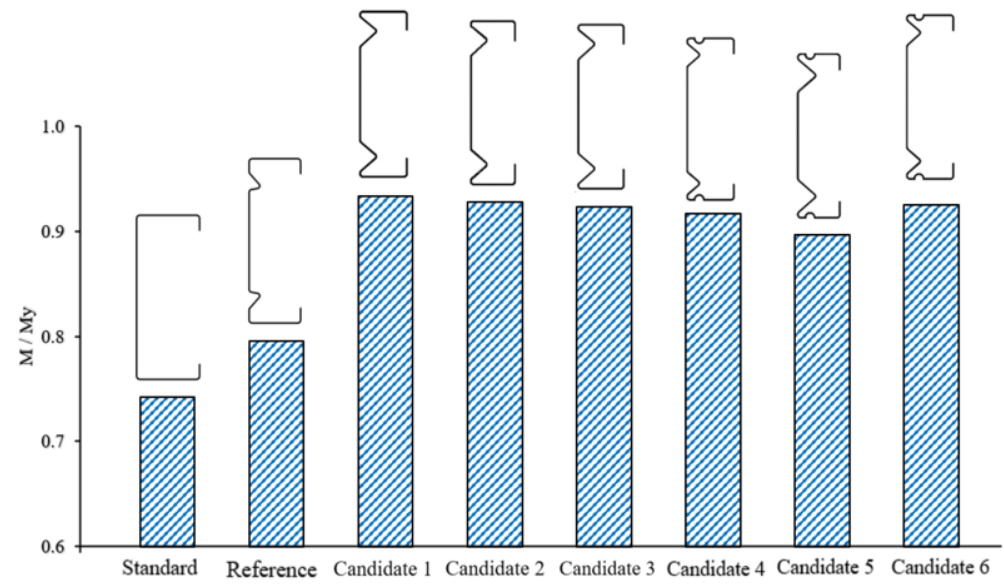

Figure 8. Results of standard, reference, and optimized channel sections for ultimate moment capacity [8].

\section{Conclusion}

This paper has provided a brief presentation of using FE simulation techniques for new process and product developments in metal forming industry. Provided industrial examples include the simulation of a novel Ultra STEEL ${ }^{\circledR}$ dimpling process and optimization of cold rolled forming products. They demonstrate that the FE modelling together with optimization techniques provide powerful tools to analyze complex processes and products. The successful simulations enable metal forming industry (Hadley Industries plc) to provide novel Ultra STEEL ${ }^{\circledR}$ products or alternative plain products with stiffeners which are thinner and therefore more competitive.

\section{References}

[1] M.A. Castellucci, I. Pillinger, P. Hartley, G.T. Deeley, The optimisation of cold rolled formed products, Thin-Walled Structures 29 (1997), 159-174.

[2] V.B. Nguyen, C.J. Wang, D.J. Mynors, M.A. English, M.A. Castellucci, Mechanical properties and structural behaviour of cold-rolled formed dimpled steel, Steel Research International, Special Issue (2011), 1072-1077.

[3] V.B. Nguyen, C.J. Wang, D.J. Mynors, M.A. English, M.A. Castellucci, Compression tests of cold-formed plain and dimpled steel columns, Journal of Constructional Steel Research 69 (2012), 20-29.

[4] V.B. Nguyen, C.J. Wang, D.J. Mynors, M.A. Castellucci, M.A. English, Finite Element simulation on mechanical and structural properties of cold-formed dimpled steel, Thin-Walled Structures, 64 (2013), $13-22$.

[5] V.B. Nguyen, C.J. Wang, D.J. Mynors, M.A. Castellucci, M.A. English, Dimpling process in cold roll metal forming by finite element modelling and experimental validation, Journal of Manufacturing Processes 16 (2014), 363-372.

[6] V.B. Nguyen, C.H. Pham, B. Cartwright, M. English, Design of new cold rolled purlins by experimental testing and Direct Strength Method, Thin-Walled Structures 118 (2017) 105-112.

[7] V.B. Nguyen, M. English, The optimization of thin-walled cold rolled products using Finite Element modelling and Design Of Experiments, The 8th International Conference on Thin-Walled Structures (2018), Lisbon, Portugal.

[8] S. Qadir, V.B. Nguyen, I. Hajirasouliha, B. Cartwright, M. English, Optimal design of cold roll formed steel channel sections under bending considering both geometry and cold working effects, Thin-Walled Structures 157 (2020) 107020. 